\title{
Quantitative Study of Myocardial Microcirculation in Arterial Hypertension due to Progressive Inhibition of NO Synthesis
}

\author{
Leila Maria Meirelles Pereira, Carlos A lberto Mandarim-de-Lacerda
}

Rio de Janeiro, RJ - Brazil

Objective - To study the quantitative changes in intramyocardial blood vessels in rats in whom nitric oxide synthesis was inhibited.

Methods - Four groups of 10 rats were studied: control (C25 and C4O) and L-NAME (L25 and L4O). The animals $L 25$ and $L 40$ received $L-N A M E$ in the dosage of $50 \mathrm{mg} / \mathrm{kg} /$ day for 25 and 40 days, respectively. On days 26 and 41 the animals in groups 25 and 40 were sacrificed. Analysis of the myocardium was performed using light microscopy and stereology.

Results - Arterial blood pressure and heart weight increased 74.5 and $57.8 \%$ after 25 days and 90.2 and $34.6 \%$ after 40 days, respectively. Comparing the L-NAME rats with the respective controls revealed that vessel volume density decreased $31.3 \%$ after 40 days, and the vessel length-density decreased $53.5 \%$ after 25 days and $25.7 \%$ after 40 days. The mean cross-sectional area of the vessels showed an important reduction of $154.6 \%$ after 25 days. The intramyocardial vessels decreased significantly in lengthdensity in the L-NAME animals. The mean cross-sectional area of the vessels, which normally increases during heart growth between 25 and 40 days, showed a precocious increase by the $25^{\text {th }}$ day in the L-NAME rats. This suggests an increase of the size of the heart, including blood vessels.

Conclusion - The inhibition of the NO synthesis provokes rarefaction in the intramyocardial vessels that progresses with the time of administration of L-NAME.

Key words: nitric oxide, myocardium, hypertension

Rio de Janeiro State University.

Mailing address: Leila Maria M. Pereira - Centro Biomédico, Instituto de Biologia Av. 28 de Setembro, 87 (fundos) - 20551-030 - Rio de Janeiro, RJ.

Received on $12 / 14 / 98$

Accepted on 3/24/99
Adult mammalian cardiac myocytes are unable to replicate themselves ${ }^{1}$. Therefore, to resist pressure overload imposed by arterial hypertension, cardiac myocytes become hypertrophic, enlarging both inlength and diameter. Hyperplasia of the cellular components of the connective tissue follows cardiac myocyte hypertrophy, for these cells do not loose their ability to replicate themselves ${ }^{2}$. Arterial hypertension is one of the risk factors for sudden cardiac death. This risk is even higher in patients who develop left ventricular hypertrophy in response to hypertension ${ }^{3}$.

Nitric oxide (NO) is produced by vascular endothelium, and it acts as a potent vasodilator, controlling blood pressure and myocardial perfusion ${ }^{4,5}$. Endothelial cells synthesize and release NO, which in turn performs important regulatory functions for vascular tonus, platelet aggregation, leukocyte adhesion and vascular smooth muscle cell proliferation ${ }^{6}$. The activity of NO-synthase is inhibited by L-arginine ( $\mathrm{N}^{\mathrm{G}}$-nitro-L-arginine-methyl-ester; L-NAME) ${ }^{7,8}$ and this inhibition represents a very useful model for studying experimental hypertension ${ }^{9}$.

Administration of L-NAME to rats promotes elevation of blood pressure and widespread reduction of peripheral blood flow, indicating that a continuous release of NO is important for controlling vascular tonus. Therefore, NO deficiency is associated with vascular relaxation deficit and vascular wall hypercontractility ${ }^{10}$. Nitric oxide seems to be a chemotactic factor for angiogenesis and NO-synthase inhibitors like L-NAME may impair neovascularization ${ }^{11}$.

All the changes that occur in the cardiovascular system following NO-synthase inhibition and myocardial hypertrophy (as a result of systemic hypertension-caused NO inhibition), as well as fibrosis and coronary vascular remodeling have been reported in the medical literature ${ }^{12-14}$.

A chronic increase in shear stress releases NO, inducing vasodilation and an increase in the vascular luminal diameter, normalizing wall stress. According to Laplace's law, vascular enlargement is associated with increasing wall stress that can stimulate protein synthesis in the extracellu- 
lar matrix and produce smooth muscle cell hypertrophy. This vascular remodeling does not normalize wall stress but leads to recovery of dynamic mechanical properties and to adaptation of arterial dimensions to enhance blood flow ${ }^{15}$.

Cardiac hypertrophy due to pressure overload also modifies the vascular structure in other organs and promotes changes in the extracellular space ${ }^{16}$. Myocyte degeneration and fibrosis occur in advanced stages of cardiac hypertrophy, because oxygen demand increases in the hypertrophic ventricle, exceeding the capability of vessels to supply adequate blood flow ${ }^{17}$.

Myocardial microcirculatory vessels are adjacent to cardiac myocytes keeping a mean distance of $8 \mu \mathrm{m}$. This short distance suggests that vasoactive substances released by microvascular endothelium can affect the adjacentmyocardium ${ }^{18}$.

Recent studies in rats have demonstrated that the chronic administration of NO-synthase inhibitors can cause hypertension, reducing intracellular levels of GMP cyclic in smooth muscle cells, promoting structural modifications in microvascular circulation ${ }^{12}$.

\section{Methods}

Forty normotensive adult male rats were used in this study (Wistar, body weight of $250 \pm 36 \mathrm{~g}$, and initial blood pressure under $120 \mathrm{mmHg}$ ). For two weeks the animals were kept under observation and underwent weekly blood pressure determinations. After this initial observation period, the animals were divided into four groups housed under appropriate conditions of light and temperature.

Groups were separated as follows: a) 25-day control (C25); b) 40-day control (C40); c)L-NAME 25 days (L25); d) L-NAME40 days (L40).

Animals in groups $\mathrm{C} 25$ and $\mathrm{C} 40$ received water and food ad libitum. Animals in groups L25 and L40 received LNAME ( ${ }^{\mathrm{G}}$-nitro-L-arginine-methyl-ester, $\mathrm{HCl}$, Sigma Co. batch $44: 0102,50 \mathrm{mg} / \mathrm{Kg}$ per day) for 25 and 40 days, respectively [13]. This investigation was performed in accordance with the Guidelines for Care and Use of Laboratory Animals published by the United States National Institutes of Health (NIHPublication \#85-23, revised in 1985).

At the end of the treatment period $\left(26^{\text {th }}\right.$ day for groups $\mathrm{C} 25$ and L25 and $41^{\text {st }}$ day for groups C40 and L40) the rats were anesthetized with inhaled ether and killed with an intracardiac injection of $10 \%$ potassium chloride, which induced a diastolic heart arrest. The weights and volumes of the hearts were measured (Scheler's method, hanging the hearts on a string and immersing them in a saline solution over an analytical precision scale) ${ }^{19,20}$. Afterwards, the hearts were immersion-fixed in a $4 \%$ formalin buffered phosphate solution ( $\mathrm{pH}$ 7.2) for 48 hours.

The myocardium is an anisotropic tissue, but isotropic sections are needed to perform stereological studies, and for this reason the hearts were prepared in accordance with the orthtopic method ${ }^{14}$. The material was embedded in paraffin, cut in $5 \mu \mathrm{m}$-thick slices and stained with hematoxylin-eosin and Picro-sirius. A stereological analysis was performed in 15 random microscopic fields using an immersion lens, Leica microscopy video camera (model DMRBE), and test-system M42 calibrated to $1 / 100 \mathrm{~mm}^{2}$ eitz micrometer ${ }^{19}$.

The volume-density of intramyocardial vessels ( $\mathrm{Vv}$ [vessels]) was determined by counting methods and calculated as: (Pp [vessels] / pt) \% where Pp [vessels] is the number of points over the vessels and $P t$ is the total number of test points, in this case 42 points.

The length-density of intramyocardial vessels (Lv[vessels]) was determined by:

$\mathrm{Lv}$ [vasos] $=2 . \mathrm{Q}_{\mathrm{A}} \mathrm{mm} / \mathrm{mm}^{3}$ where $Q a$ is the numerical vascular density by the myocardial area.

The cross-sectional area of vessels was assessed by:$\left[\mathrm{Aa} / \mathrm{Qa} \mathrm{mm} \mathrm{mm}^{2}\right]$ where $A a$ is the density of blood vessels by area.

\section{Results}

In rats from the control groups cardiac myocytes and intramyocardial vessels remained unchanged after 25 and 40 days. Rats that underwent NO-synthase inhibition had myocardial remodeling compatible with this model of arterial hypertension, showing perivascular and interstitial fibrosis and cardiac myocyte hypertrophy. These lesions were more intense as the time of NO inhibition increased (figures 1 to 4 ).

When compared with control animals, arterial blood

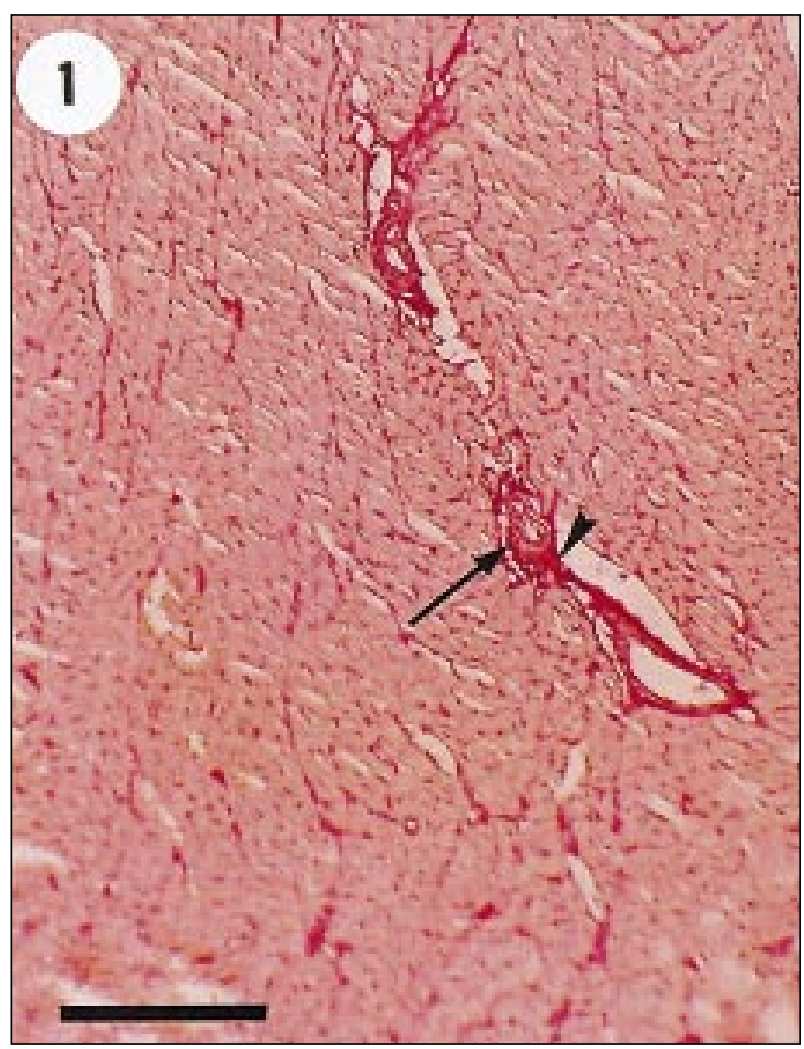

Fig. 1 - Rat myocardium after exposure to L-NAME for 25 days. Note mild tissue disorganization and the small arteries (arrow) presenting with perivascular fibrosis (arrow head). H-E (bar=280 $\mu \mathrm{m})$ 


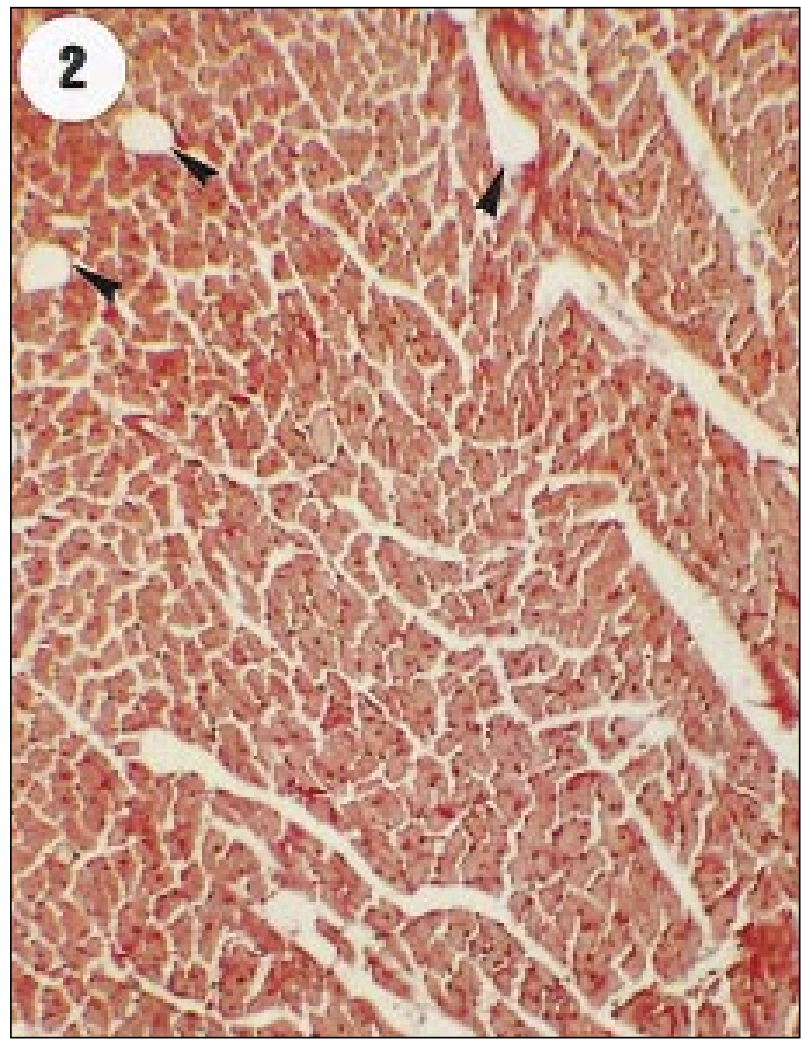

Fig. 2-Photomicrography of control rat myocardium after 25 days. The myocardial tissue is well organized and the vessels have no lesions (arrow head). H-E (bar=280um) pressure was $74.5 \%$ higher on the $25^{\text {th }}$ day and $90.2 \%$ higher on the $40^{\text {th }}$ day. Cardiac weight increased $57.8 \%$ in L25 rats and $34.6 \%$ in $\mathrm{L} 40$ rats when compared with their respective controls (fig. 5).

Significant stereological differences were revealed between control and L-NAME rats as shown in figures 2 to 4. Compared with their respective controls, $\mathrm{Vv}$ [vessels] decreased by $31.3 \%$ in rats from group L 40 (fig. 6, median percent variation).

$\mathrm{Lv}$ [vessels] decreased $53.5 \%$ in group $\mathrm{L} 25$ and $25.7 \%$ in L40. However between groups $\mathrm{C} 25$ and $\mathrm{C} 40$ a decrease of $52.2 \%$ occurred in Lv[vessels] (fig. 7, median percent variation).

$\mathrm{Aa}$ [vessels] increased in two situations. It was $154.6 \%$ higher in L25 animals when compared with C25 animals, and it was also $198 \%$ higher in C40 animals when compared with C25 animals (fig. 8, median percent variation).

\section{Discussion}

The capillary to myocardial ratio in the normal adult myocardium is $1: 1$. In cardiac hypertrophy, the number of capillaries in the myocardium remains the same but the myocytes are enlarged, resulting in relative ischemia that is proportional to the degree of hypertrophy ${ }^{21}$. In addition, evidence exists that $\mathrm{NO}$ acts as a chemotactic factor for angiogenesis and that the inhibition of NO production impairs neovascularization ${ }^{11}$.

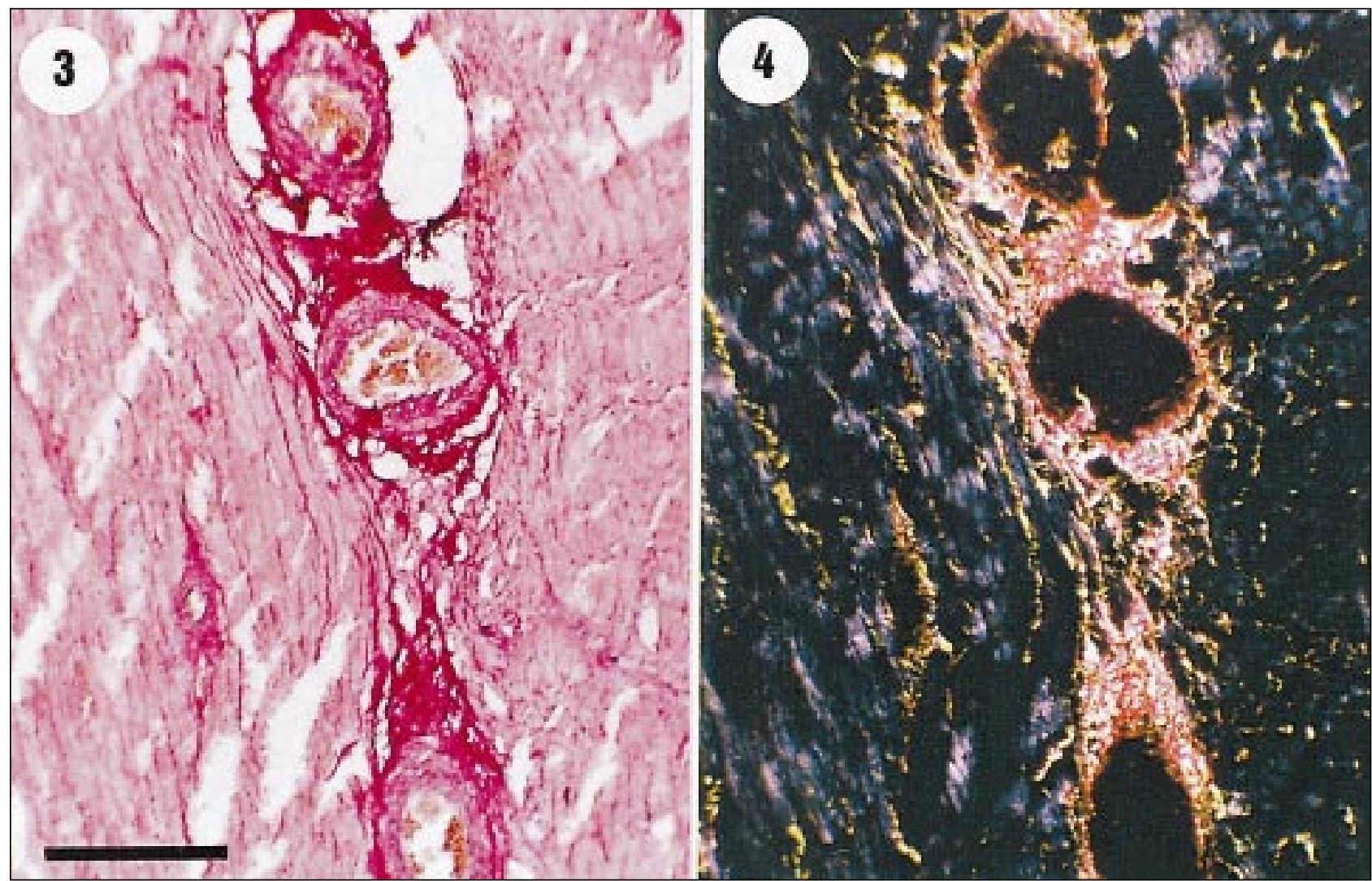

Figs. 3 and 4-Photomicrographies of a hipertensive rat that received L-NAME for 40 days. Note the intense perivascular fibrosis and thickning of the vessel wall. Picro-Sirius (bar= $85 \mu \mathrm{m}$ ). 


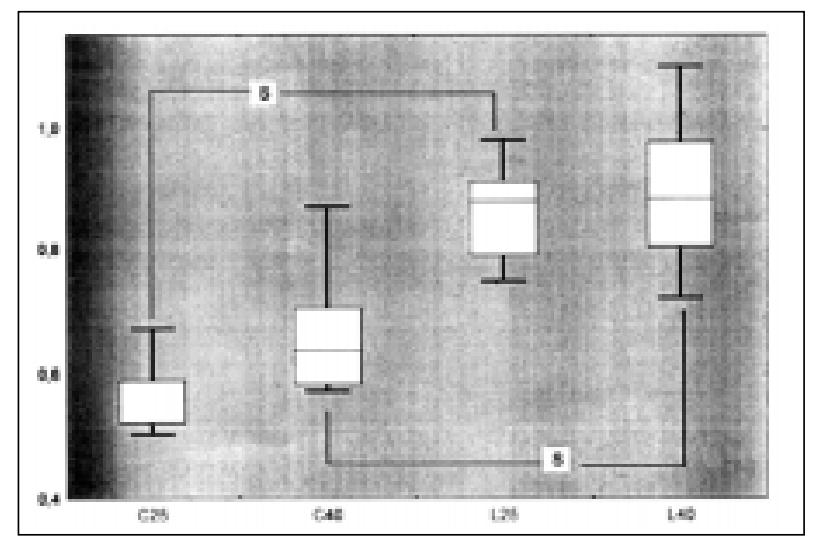

Fig. 5 - Box plot of the cardiac weight in the four groups of animals. C25 - control rats 25 days; $\mathrm{C} 40$ - control rats 40 days; $\mathrm{L} 25$ - L-NAME for 25 days; L40 - L-NAME for 40 days. $S=p<0.05$.

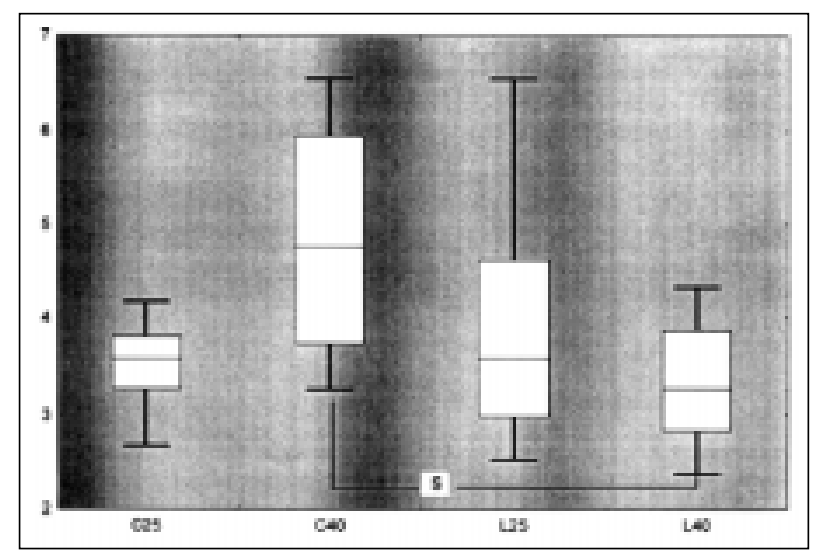

Fig. 6-Box plot of the volume density of the intramyocardial vessels.

In our study, the myocardium of animals that received L-NAME showed morphological modifications that resulted from the extension of time of NO synthesis inhibition. In this model of arterial hypertension there are descriptions of large areas of interstitial fibrosis mostly in endomysium and perimysium and also perivascular fibrosis around intramyocardial coronary branches and areas of ischemic lesions with inflammatory infiltrate. The intimal layer of small intramyocardial arteries is normally thick ${ }^{13,14}$. Administration of LNAME at $12 \mathrm{mg} / \mathrm{kg}$ per day for 15 weeks (105 days) caused myocyte hypertrophy and little interstitial and perivascular fibrosis; however, no significant quantitative modifications in microcirculation occurred ${ }^{22}$. In the present study, in which L-NAME was administered as daily doses of $50 \mathrm{mg} / \mathrm{kg}$ for 25 or 40 days, we observed marked interstitial and perivascular fibrosis with significant modifications of myocardial microcirculation. These findings indicate that the modifications present in this model are dependent on the time and dose of NO inhibitor administration.

The present study revealed an important diminution in the length-density of myocardial vessels in rats that underwent NO inhibition for 25 and 40 days. Moreover, the volume-density of vessels also diminished in these animals after 40 days. The cross-sectional area of intramyocardial

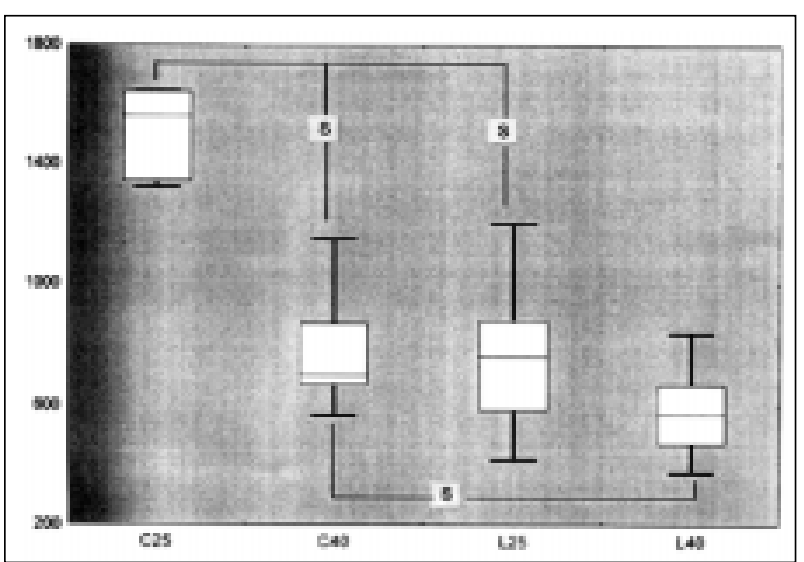

Fig. 7 - Box plot of the lengh density of the intra myocardial vessels.

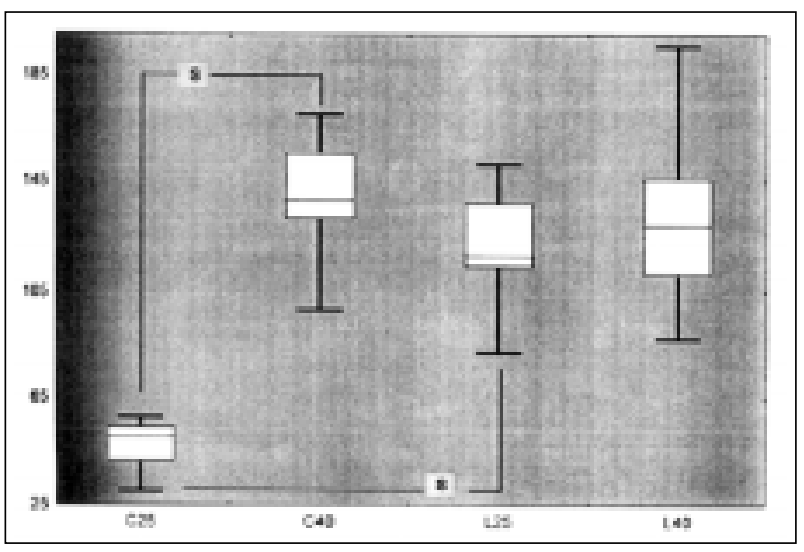

Fig. 8-Box plot of the cross sectional area of the intra myocardial vessels.

vessels that normally increases during cardiac growth between the $25^{\text {th }}$ and $40^{\text {th }}$ days, increased earlier in animals that underwent NO inhibition after 25 days, suggesting that these rats had an early enlargement of the heart (including vessels) due to pressure overload.

Capillary network increase parallel to the increase of the cross-sectional area of the muscular cells has been reported $^{23,24}$. In a previous study, we observed a significant increase in the cross-sectional area of cardiac myocytes after 25 and 40 days of NO inhibition ${ }^{14}$. This factor may be responsible for the significant increase in cardiac weight at 25 and 40 days. The increment in cardiac weight was higher at 25 days then at 40 days in animals that received L-NAME, compared with their respective controls. It is known that the size of the heart is related to its collagen content and the size of the muscular cell ${ }^{25,26}$. Therefore, we believe that 25 days of ventricular overload is enough to induce significant myocyte hypertrophy (explaining the increased weight). Nevertheless, if the overload is extended for a few more days (i.e., 40 days), the increment in cardiac weight is less marked because it is produced by connective tissue deposition (characterized by an increase in cardiac interstitium volume-density).

A recently published study shows that cardiac hyper- 
trophy induced by angiotensin II in rats results in an $18 \%$ reduction in capillary density and a 54\% increase in arteriolar density. Previous treatment with angiotensin antagonists (receptor subtypes 1 and 2) prevented an increase in arteriolar density, but only losartan prevented changes in capillary density. These findings suggest that angiotensin II dependent cardiac hypertrophy is associated with capillary rarefaction and arteriolar development and that these two processes are regulated independently ${ }^{27}$. Recent reports have suggested that angiotensin II may play an important role in cardiac hypertrophy, acting on muscle cells and fibroblasts both from the myocardium and vessels through insulin-like growth factor 1 that leads to myocyte hypertrophy and to a progressive deposit of collagen in the perivascular and interstitial space. Angiotensin II blocks the enzymatic action of collagenase that promotes the turnover of collagen and, because of that, the myocardial and vascular tissues may become more rigid ${ }^{28,29}$.

In the present study, we analyzed stereologically the intramyocardial capillaries and arterioles in conjunction, using an experimental model for which the complete understanding is yet unknown but is probably angiotensindependent. Because of this uncertainty, new studies are needed to better clarify the consequences of $\mathrm{NO}$ action and inhibition of myocardial physiology.

The beginning and progression of myocyte necrosis may be related to functional and structural abnormalities in the vascular network ${ }^{30}$. The more that cardiac hypertrophy develops, the more heterogeneous is myocyte size and the less homogeneous is intercapillary distance, which may explain the ischemic necrosis that follows the imbalance of oxygen supply and demand ${ }^{31}$. There are several reports of extensive areas of fibrosis, necrosis and granulation tissue in models of arterial hypertension induced by NO inhibition ${ }^{32}$. Our results are in accordance to these reports.

\section{Acknowledgment}

This research was funded in part by CNPq (grant 57.41.14/97-1) and Faperj (grantE-26/170.086/97).

\section{References}

1. Mandarim-de-Lacerda CA, Santos MB, Le Floch-Prigent P, Narcy F. Stereology of the myocardium in human foetuses. Early Hum Develop 1997; 48: 249-59.

2. Zak R. Cell proliferation during cardiac growth. Am J Cardiol 1973; 31:211-9.

3. Schillaci G, Verdecchia P, Borgroni C, et al. Association between persistent pressure overload and ventricular arrhythmia in essential hypertension. Hypertension 1996; 28: 284-9.

4. Moncada S, Higgs EA. Endogenous nitric oxide inhibition as a model of hypertensive heart muscle disease. Eur J Clin Invest 1991; 21: 361-74.

5. Suto N, Mikuniya A, Okubo T, Hanada H, Shinozaki N, Okumura K. Nitric oxide modulates cardiac contractility and oxygen consumption without changing contractile efficiency. Am J Physiol 1998; 275: H41-9.

6. Arnal JF, Armani EI, Chatellier G, Menard J, Michel JB. Cardiac weight in hypertension induced by nitric oxide synthase blockade. Hypertension 1993; 22: 380-7.

7. Gardiner SM, Compton AM, Bennet T, Palmer RMJ, Moncada S. Regional haemodinamic changes during oral ingestion of $\mathrm{N}^{\mathrm{G}}$-monomethyl-L-arginine or $\mathrm{N}^{\mathrm{G}}$-nitro-L-arginine methyl ester in conscious Brattleboro rats. Br J Pharmacol 1990; 101: 10-2

8. Palmer RMJ, Ashton DS, Moncada S. Vascular endothelial cells synthesize nitric oxide from L-arginine. Nature 1988; 333: 664-6.

9. Pechanova O, Bertanova I, Pelouch V, Simko F. Protein remodeling of the heart in NO-deficient hypertension: the effect of Captopril. J Moll Cell Cardiol 1997; 29 3365-74.

10. Holecyova A, Torok J, Bernatova I, Pechanova O. Restriction of nitric oxide rather than elevated blood pressure is responsible for alterations of vascular responses in nitric oxide-deficient hypertension. Physiol Res 1996; 45: 317-21.

11. GranderHJ,Ziche M,HawkerJR, MeiningerCJ,CzisnyLE,Zawieja DC. Molecular and cellular basis of myocardial angiogenesis. Cell Mol Biol Res 1994; 40: 81-5.

12. Numaguchi K, Egashira K, Takemoto M, et al. Chronic inhibition of nitric oxide synthesis cause coronary microvascular remodeling in rats. Hypertension 1995; 26(part 1): 957-62.

13. Pereira LMM, Mandarim-de-Lacerda CA. Quantitative examination of the cardiac myocytes in hypertensive rats under chronic inhibition of nitric oxide synthesis. J Biom Sci 1998; 5: 363-9.

14. Pereira LMM, Vianna GMM, Mandarim-de-Lacerda CA. Stereology of the myocardium in hypertensive rats. Differences in relation to the time of inhibition of nitric oxide synthesis. Virchows Arch 1998; 433: 369-73.

15. Driss AB, Benessiano J, Poitevin P, Levy BY, Michel JB. Arterial expansive remodeling induced by high flow rates. Am J Physiol 1997; 272: H851-8.

16. Ito N, Nitta $\mathrm{Y}$, Ohtani H, Ooshima A, Isoyama S. Remodeling of microvessels by coronary hypertension or cardiac hypertrophy in rats. J Moll Cell Cardiol 1994; 26: 49-59.
17. Buja LM, Muntz KH, Lipscomb K, Willerson JT. Cardiac hypertrophy in chronic ischemic heart disease. In: Tarazi R (ed) - Mechanisms of Left Ventricular Hypertrophy. New York: Raven Press, 1983: 287-94.

18. Xie YW, Shen W, Zhao G, Xu X, Wolin MS, Hintze TH. Role of endothelium-derived nitric oxide in the modulation of canine myocardial mitochondrial respiration in vitro. Circ Res 1996; 79: 381-7.

19. Mandarim-de-Lacerda CA. Stereology in the normal and pathological morphologic research. Biomed Res 1998; 9: 153-63.

20. Scherle WA. A simple method for volumetry of organs in quantitative stereology. Mikroskopie 1970; 26: 57-60.

21. Roberts JT. Arteries, veins and lymphatic vessels of the heart. In: Luisada AA (ed) Development and Structure of the Cardiovascular System. New York: McGrawHill, 1961: 85-118.

22. Pessanha MG, Mandarim-de-Lacerda CA, Hahn MD. Stereology and immunohistochemistry of the myocardium in experimental hypertension: long-term and low dosage administration of inhibitor of the nitric oxide synthesis. Pathobiology $1999 ; 67: 26-33$.

23. Rakusan K, Flanagan MF, Geva T, Southern J, Van Praagh R. Morphometry of human coronary capillaries during normal growth and the effect of age in left ventricular pressure-overload hypertrophy. Circulation 1992; 86: 38-46.

24. Sladek T, Gerova M, Znojil V, Devat L. Morphometric characteristics of cardiac hypertrophy induced by long-term inhibition of NO synthase. Physiol Res 1996; 45: 335-8.

25. Contard f, Koteliansky V, Marotte F, Dubus I, Rappaport L, Samuel JL. Specific alteration in the distribution of extracellular matrix components within rat myocardium during the development of pressure overload. Lab Invest 1991; 64: 65-75.

26. Zhang L, Summers KM, West MJ. Analysis of linkage of the ACE locus with measures of cardiac hypertrophy in the spontaneously hypertensive rat. Clin Exp Pharmacol Physiol 1996; 23: 597-9.

27. Sabri A, Samuel J-L, Marotte F, Poitevin P, Rappaport L, Levy BI. Microvasculature in Angiotensin II-dependent cardiac hypertrophy in the rat. Hypertension 1998; 32: 371-5.

28. Weber KT, Brilla CG. Pathological hypertrophy and cardiac interstitium. Circulation 1991; 83: 1849-65.

29. Wilke A, Funk R, Rupp H, Brilla CG. Effect of the renin-angiotensin-aldosterone system on the cardiac interstitium in heart failure. Basi Res Cardiol 1996: 91(suppl 2): 79-84.

30. Hachamowiitch R, Wicker P, Capasso JM, Anversa P. Alterations of coronary blood flow and reserve with aging in Fischer 344 rats. Am J Physiol 1989; 256 H66- H72.

31. Suzuki Y, Harada K, Kawamura K, Matsuda H, Tarada G. Limited adaptation in 
Pereira \& Lacerda

Inhibition of NO sinthesis in myocardial microcirculation
Arq Bras Cardiol volume 73, ( n $^{\circ}$ 5), 1999 chronically hypertrophied hearts from aortic constricted rats: increased inhomogeneity in cross sectional area of cadiomyocytes and intercapillary distance. Tohuku J Exp Med 1993; 170: 181-95.
32. Babál P, Pechánová O, Bernátová I, Stvrtina S. Chronic inhibition of NO synthesis produces myocardial fibrosis and arterial media hyperplasia. Histol Histopathol 1997; 12: 623-9. 